Review Article

\title{
Functional Hydrogels and Their Application in Drug Delivery, Biosensors, and Tissue Engineering
}

\author{
Ke Wang $\left(\mathbb{D},{ }^{1}\right.$ Yuting Hao, ${ }^{2}$ Yingna Wang, ${ }^{3}$ Jinyuan Chen, ${ }^{2}$ Lianzhi Mao, ${ }^{2}$ Yudi Deng, ${ }^{2}$ \\ Junlin Chen, ${ }^{2}$ Sijie Yuan, ${ }^{4}$ Tiantian Zhang $\left({ }^{5},{ }^{5}\right.$ Jiaoyan Ren $\oplus^{1},{ }^{1}$ and Wenzhen Liao $\mathbb{D}^{2}$ \\ ${ }^{1}$ College of Light Industry and Food Sciences, South China University of Technology, Guangzhou 510640, China \\ ${ }^{2}$ Department of Nutrition and Food Hygiene, Guangdong Provincial Key Laboratory of Tropical Disease Research, School of \\ Public Health, Southern Medical University, No. 1023 South Shatai Road, Guangzhou 510515, China \\ ${ }^{3}$ Guangzhou Sanxing Biotechnology Co. Ltd., No. 14, Shayuan Shang Street, Sixian Village, Xinzhuang Town, Panyu District, \\ Guangzhou 511436, China \\ ${ }^{4}$ Department of Endocrinology, The Third Affiliated Hospital, Southern Medical University, Guangzhou 510630, China \\ ${ }^{5}$ College of Food Science and Engineering, Ocean University of China, No.5 Yushan Road, Qingdao 266003, China
}

Correspondence should be addressed to Tiantian Zhang; ttzhang89@hotmail.com, Jiaoyan Ren; jyren@scut.edu.cn, and Wenzhen Liao; wenzhenliao@163.com

Received 23 May 2019; Accepted 6 July 2019; Published 7 October 2019

Guest Editor: Lin Zhang

Copyright ( $) 2019$ Ke Wang et al. This is an open access article distributed under the Creative Commons Attribution License, which permits unrestricted use, distribution, and reproduction in any medium, provided the original work is properly cited.

\begin{abstract}
Hydrogel is a new class of functional polymer materials with a promising potential in the biomedical field. The purpose of this article is to review recent advancements in several types of biomedical hydrogels, including conductive hydrogels, injectable hydrogels, double network hydrogels, responsive hydrogels, nanocomposite hydrogels, and sliding hydrogels. In comparison with traditional hydrogels, these advanced hydrogels exhibit significant advantages in structure, mechanical properties, and applications. The article focuses on different methods used to prepare advanced biomedical hydrogels and their diversified applications as drug delivery systems, wound dressings, biosensors, contact lenses, and tissue replacement. These advances are rapidly overcoming current limitations of hydrogels, and we anticipate that further research will lead to the development of advanced hydrogels with ubiquitous roles in biomedicine and tissue replacement and regeneration.
\end{abstract}

\section{Introduction}

Hydrogels are a class of polymers having a three-dimensional network structure formed through physical and chemical cross-linking of monomers with a hydrophilic group [1]. Hydrogels swell when they absorb large volumes of water yet maintain their original structures without being dissolved [2, 3] (Figure 1). In the biomedical field, hydrogels are a new class of functional polymer materials with enormous potential in biotechnology. When the polymer network is encased in water, the material absorbs the water and adopts fluidic properties, which is very similar to what occurs with tissues in the human body $[4,5]$. In the presence of water, the surfaces of the hydrogels become wet and malleable. And because of these properties, couple with the stable organization of the material significantly reduces irritation to the surrounding tissues and improves biocompatibility [6]. Additionally, hydrogels will not affect the metabolic processes of living organisms and metabolites can pass freely through the hydrogels. Hydrogels are also sensitive to small changes in external stimuli, such as temperature, $\mathrm{pH}$, ionic strength, electric fields, and magnetic fields, and can respond to these stimuli through volume swelling or shrinking [7-11]. Therefore, hydrogels are more similar to living tissues, specifically the outer membrane of the cell matrix, than any other currently known synthetic biomaterials and these properties result in reducing friction and mechanical effects on the surrounding tissues, which significantly improves the biological properties of the material [12].

Hydrogels have been one of the greatest interests to biomaterial scientists since the pioneering work of Wichterle 


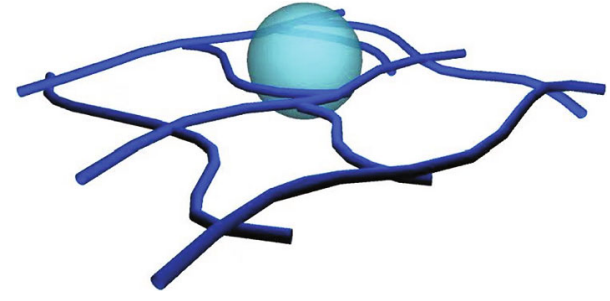

FIGURE 1: The sketch map of the hydrogel network.

and Lim in 1960 on cross-linked HEMA (hydroxyethyl methacrylate) hydrogels, which has led to the development of materials with excellent water absorption, high water retention, good biocompatibility, biodegradability, limited or minimal toxicity, and simplified synthesis methods. These materials are now widely used in biomedicine, functioning as tissue fillers, drug delivery agents, contact lenses, and tissue engineering scaffold materials [12-14]. Because hydrogels produce huge economic and social benefits, the research, the development, the application, and the production of hydrogels represent an important area of the biomaterial field.

Traditional hydrogels are typically formed by chemical cross-linking. Nonuniform dispersion of a chemical crosslinking agent results in a nonuniform gel network, and the resulting gel is very weak and fragile, greatly limits its application [15]. To overcome mechanical limitations in traditional gels, four major types of novel network structures have been investigated, as follows: (i) the replacement of covalent cross-linking points by active cross-linking sites, which can reduce the stress concentration and the network structural damage caused by the uneven distribution of covalent bonds. Representative studies include "Slide-Ring" hydrogels, in which " 8 "-shaped polyrotaxane as a crosslinking point, polymer chains pass through the cross-linking agent from its upper and lower cavities [16]. The molecular chains distribute stress more uniformly across the gel network through positional adjustment in the external force. (ii) The introduction of a "sacrifice key" within the structure of certain hydrogels (e.g., double network hydrogels), which is a region designed to break and absorb energy that can improve the strength and resilience of the materials [17]. These hydrogels are mainly composed of two interpenetrating or semi-interpenetrating networks with different properties. Under stress, the first network absorbs energy to burst, endowing hydrogels with high strength and toughness, while the second network is loosely cross-linked and is more difficult to destroy, which maintains the integrity of hydrogels [3]. (iii) Nanoparticles as giant multifunctional crosslinking points, where polymer chains are cross-linked into a three-dimensional network by physical adsorption or chemical bonding [18]. The physical adsorption between the molecular chains and nanoparticles can dissipate energy to improve the mechanical properties of hydrogels, while conversely, the high specific surface area and high modulus of nanoparticles themselves strengthen the hydrogels [19]. (iv) Polymers constructed via noncovalent interactions and supramolecular self-assembling structures, producing hydrogels that are strong, resilient, and responsive to changing stimuli [20]. Consequently, the objective of this review is to evaluate recent research and progress aimed at developing novel high-performance, intelligent hydrogel materials for biomedical applications.

\section{Main Types of Hydrogels}

2.1. Conductive Hydrogels. Conductive hydrogel $(\mathrm{CH})$ is a new effective material which has the similar unique properties to traditional hydrogels and an additional benefit in electrical conductivity [21-23]. It was first proposed by Gilmore et al. [24], and in recent years, more attention has been given in the exploitation and application of CHs. Depending on the different additives, the $\mathrm{CHs}$ are divided into two categories: (i) the CHs-based conducting polymers (CPs) and (ii) the $\mathrm{CHs}$-based metallic nanoparticles. Mostly, the $\mathrm{CH}$ is defined as a hybrid network made by cross-linked soft hydrogels and $\mathrm{CPs}$ [25]. Although $\mathrm{CP}$ is not metallic, it has extraordinary electrical conductivity that can change the final hydrogels in structural and electrical properties to a considerable extent [26-31]. The use of CPs enables hydrogels to stimulate electricity locally and enhance the physical properties of hydrogels to accurately control the extent and duration of external stimulation $[32,33]$. The microscopy allows the microstructure magnified, and it always be used to observe the microstructure of the hydrogel, such as atomic force microscopy, which can not only observe the microstructure of objects $[34,35]$ but also the conductivity of the conductive hydrogel that can be detected by conducting probe atomic force microscopy [36]. Nowadays, the most familiar CPs like polypyrrole (PPY), polythiophene (PT), poly(3,4-ethylene dioxythiophene) (PEDOT), and polyaniline (PAni) are widely used in biomedical science to promote cell growth and proliferation [37, 38]. Besides, the natural polymers such as alginate, starch, chitosan, and their derivatives also have been considered as CPs in different conditions and because of the characteristics of these natural polymers, the final hydrogels are extremely beneficial to biodegradable and biocompatible. Till now, the CPs has gained lots of scientific responsiveness and they have extensively applied in batteries, sensors, semiconductor devices, electronic and optoelectronic devices, and so on [39-43]. Similar to the CPs mentioned before, the metallic nanoparticles give the $\mathrm{CH}$ synergistic properties combined with the metal and hydrogel matrix $[44,45]$. And since the performance of the hydrogels has improved the homogeneous distribution and long-term cytotoxicity of metallic nanoparticles, more applications of final hydrogels are being developed in the field of biomaterials.

A number of routes have explored to the preparation of $\mathrm{CHs}$, but all of them can be summarized as two types: preparation of single component $\mathrm{CHs}$ and preparation of multicomponent CHs. Single-component $\mathrm{CHs}$ are defined as a stable conjugate-combined conductive polymer with small hydrophilic molecule through electrostatic interaction or cross-linked by substitutes of small molecular. According to the electrostatic interaction between the positive charges on PAni with the phosphate on phytic acid, the final hydro-

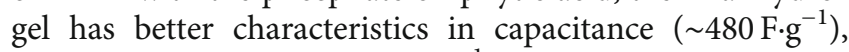
higher sensitivity $\left(\sim 16.7 \mu \mathrm{A} \cdot \mathrm{mM}^{-1}\right)$, and faster response time $(\sim 0.3 \mathrm{~s})$ in the glucose sensing test [46]. The common 
methods to prepare multicomponent $\mathrm{CHs}$ are electrochemical polymerization and chemical oxidation polymerization; the latter usually includes one-step facile strategy and twostep sequential preparation method. The one-step facile strategy refers to the cross-linked hydrogels obtained by the chemical reaction of CPs and hydrophilic polymer mixture. PPY has poor water solubility, but the PPY/agarose $\mathrm{CH}$ can get by this route when PPY is added to aqueous solution of agarose and cupric chloride is added to the solution to oxidize PPY $[47,48]$. The two-step sequential preparation method is more complex; the extra step is the monomers of CPs which permeate into hydrophilic polymer hydrogels. The preparation of PPY conductive hydrogel can also use the two-step method, incorporation of pyrrole monomer and silver nanoparticles first, and then oxidation of pyrrole with ferric ion, and it is surprising to find that the final $\mathrm{CHs}$ are beneficial to thermostability, uniform mesh structure, and mechanical property (storage modulus $10 \mathrm{kPa}$ ) [49]. Generally, because a one-step facile strategy has the advantages of simple operation, energy saving, and environmental protection while the two-step sequential preparation method needs the step of incorporation because the final hydrogels are not uniform, the former will be the main research direction of the $\mathrm{CH}$ preparation in the future.

2.2. Injectable Hydrogels. Injectable hydrogels are characterized by an intrinsic fluidity and can be applied by an injection method. In response to external stimuli (e.g., temperature or $\mathrm{pH}$ changes), injectable hydrogels exhibit sol-gel phase transitions [50]. Moreover, compared with conventional hydrogels, injectable hydrogels are more effective for minimally invasive applications. This not only expands the scope of its application in the biomedical field but also improves the comfort satisfaction of patients and reduces the cost of application to a certain extent $[51,52]$. Based on the methods used for development, injectable hydrogels can be divided into two categories: light irradiation hydrogels and selfassembling hydrogels [53, 54]. Light irradiation hydrogels involve the formation of irreversible covalent bonds through the application of visible or ultraviolet light radiation, while self-assembling hydrogels are formed spontaneously or after directional initiation $[52,55]$.

Many injectable hydrogels with different properties have been prepared using the above two gelation methods. Injectable hydrogels made of dextran methacrylate (DEX-MA) and scleroglucan $(\mathrm{Scl})$ can be formed by UV irradiation using a photoinitiator, and the resulting gels reportedly exhibited adequate mechanical properties, suitable for biomedical applications [56]. Novel injectable photochemical hydrogels are composed of gellan gum methacrylate (GG-MA) and polyethylene glycol dimethacrylate (PEG-DMA) and have also been synthesized by irradiation with a UV lamp for $30 \mathrm{~min}$ [57], and the resulting hydrogels exhibited better mechanical properties than those composed of GG-MA alone. Interestingly, the strength is significantly enhanced by the concentration and the molecular weight of PEG-DMA used to construct the new network [57]. The methods of self-assembling hydrogels include enzyme-induced gelation, chemical cross-linking with complementary groups, and ionic interactions. The injectable porous hydrogels have been widely used in biomedical applications due to their excellent permeability and ease of integration into sites of surgical intervention. For example, Yom-Tov et al. developed a method that enables the in situ formation of pores with tailored porosity and pore size, by encapsulating oil droplets in the hydrogel using an emulsion templating technique and then leaching the droplets out of the gel to create the porous structures [58]. The oil-to-water ratios and the surfactant concentrations were adjusted to vary pore size and porosity, and this method produced bioactive hydrogels exhibiting good mechanical strength, water absorbency, and diffusive properties, useful for biomedical applications [58].

2.3. Double Network Hydrogels. Double network (DN) hydrogels comprise two interpenetrating polymer networks; one of which is a highly cross-linked rigid polymer network, while the other is a lightly cross-linked flexible polymer network [59]. While this structure is connected through physical entanglement, no chemical cross-linking occurs between these two mutually independent cross-linked networks [60]. Hydrogels exhibit a certain viscous flow due to the loose cross-linking of the second network, which can effectively absorb the fracture energy through the network deformation and/or the slippage of physical entanglements along the polymer chain. This prevents cracks from propagating across the structure, so DN hydrogels have good mechanical strength [61]. In addition to greater mechanical strength, DN hydrogels have several other advantages over hydrogels with a single network structure, such as the degree of cross-linking is easier to control and increased drug loading capacity [62].

Early DN hydrogels were mainly composed of covalent bonds, but when covalent networks are destroyed by stress, hydrogels become increasingly elastic and will permanently lose their energy dissipation mechanisms [63]. In recent years, a series of DN hydrogels have been developed that introduce noncovalent (e.g., ions or hydrogen bonds) "sacrifice units" $[64,65]$ that exhibit high strength and durability $[66,67]$. The noncovalent interactions are often dynamic and reversible. When they are introduced into the hydrogels, the noncovalent "sacrifice units" may be destroyed from energy dissipation due to stress [68], then reunited to rebuild the network structure once the stressor has been removed, thus restoring the original strength and toughness of the gel [65]. For example, a novel double network hydrogel (IPN hydrogels) was fabricated by combining cellulose and poly(N-isopropyl acrylamide), in which cellulose hydrogels were employed as the first network (Figure 2(a)), while the second network was comprised of monomeric $\mathrm{N}$-isopropylacrylamide, $\mathrm{N}, \mathrm{N}^{\prime}$-methylene bis-acrylamide as a cross-linker, and ammonium persulfate as an initiator (Figure 2(b)) [69]. The two networks were subsequently integrated through a polymerization reaction at $35^{\circ} \mathrm{C}$ (Figure $2(\mathrm{c})$ ), and the resulting DN hydrogel exhibited uniform porous structure, while its mechanical and swelling properties were strongly dependent on the weight ratio of two networks [69]. In a different study, an extremely stretchable and tough hydrogel was synthesized by mixing ionically cross-linked alginate and covalently cross-linked polyacrylamide [70]. The alginate 


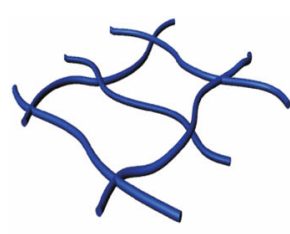

(a)

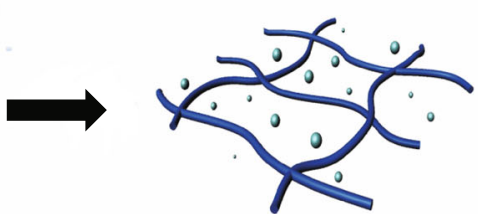

(b)

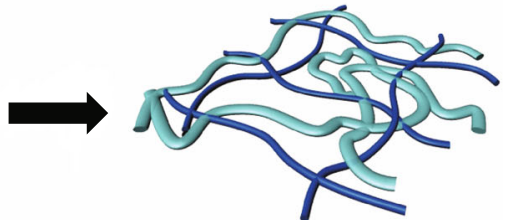

(c)

FIGURE 2: Schematic illustration of the fabrication process of IPN hydrogels.

TABLE 1: Stimulating and responsive factors of smart hydrogels.

\begin{tabular}{lcc}
\hline Type & Stimulating factors & Responsive group or substance \\
\hline Temperature-sensitive & Temperature & N-Isopropylacrylamide \\
$\mathrm{pH}-$ sensitive & $\mathrm{pH}$ & $-\mathrm{COOH}^{-}-\mathrm{HSO}_{3},-\mathrm{NH}_{2}$ \\
Light-sensitive & Light & Cinnamoyl-, azobenzene, O-nitrobenzyl alcohol \\
Electricity-sensitive & Electricity & Carbonyl-, nitro-, alkyl group \\
\hline
\end{tabular}

chain was comprised of mannuronic acid ( $M$ unit) and guluronic acid ( $\mathrm{G}$ unit) connected by glycosidic linkages. In an aqueous solution, the $\mathrm{G}$ blocks were able to chelate divalent cations (e.g., $\mathrm{Ca}^{2+}$ ) to form ionic cross-links. These initial $\mathrm{Ca}^{2+}$-cross-linked alginate hydrogels exhibited limited stretching capabilities and could rupture when stretched beyond 1.2 times its original length. However, subsequent research reported that in situ polymerization combining the $\mathrm{Ca}^{2+}$-cross-linked alginate hydrogel skeleton with an acrylamide monomer to form an interpenetrating network structure greatly improved the tensile toughness and strength of hydrogels, thus increasing its tensile breaking length up to 23 times the original length [70].

2.4. Responsive Hydrogels. Hydrogels with responsive performance have a broad range of potential applications in biological tissue engineering, including drug delivery and controlled release, artificial muscles, sensors, and enzyme catalysis [71-73]. When environmental changes (e.g., external temperature, $\mathrm{pH}$, light, electric field, and salinity) occur, hydrogels can shrink or swell as needed due to the introduction of hydrogen bonds, ions, complexation, electrostatic interactions, and other noncovalent interactions [74]. These responsive "smart" or intelligent hydrogels were first developed by Katchalsky in 1949, by copolymerizing methacrylic acid with a low percentage of divinyl benzene, producing a gel capable of absorbing hundreds of times its own weight of water at higher $\mathrm{pH}$ values, then gradually shrinking with decreasing $\mathrm{pH}$ [75]. Significant progress has been made since then, and currently, responsive hydrogels can be classified (Table 1) based on responses to different stimuli as being temperature-sensitive, $\mathrm{pH}$-sensitive, light-sensitive, or electricity-sensitive $[76,77]$.

The most common intelligent hydrogels are temperaturesensitive, particularly those comprised of $\mathrm{N}$-isopropyl acrylamide [78], where interactions between hydrophilic amide groups and hydrophobic isopropyl groups can produce structural changes in the hydrogel [79]. A novel polymer network was obtained by the introduction of the extracellular glucan salecan into a poly(N-isopropylacrylamide) (PNIPAm) network, resulting in a thermosensitive hydrogel possessing good mechanical properties and high water absorption at room temperature [80]. Moreover, the salecan/PNIPAm hydrogels were nontoxic and exhibited good biocompatibility, making them suitable for biomedical applications [80]. N,N-Diethylacrylamide has also been found to exhibit temperature sensitivity [81]. Hoffman has synthesized a series of thermo- and $\mathrm{pH}$-sensitive poly(vinyl alcohol)/poly(N,N-diethylacrylamide-co-itaconic acid) (PVA/P(DEA-co-IA)) semi-interpenetrating polymer network (semi-IPN) hydrogels by radical polymerization and semi-IPN technology [1]. The obtained semi-IPN hydrogels possessed unconventional thermosensitive properties, such as faster deswelling rates and slower swelling rates in response to an alternation of temperature, and outstanding mutative values in response to $\mathrm{pH}$ value change [1].

Smart hydrogels capable of responding to $\mathrm{pH}$ changes have also been developed by incorporating ionizable acidic or basic groups (e.g., carboxyl, sulfonic acid, or amino groups) in the preparation process [82-84]. Dissociation or association of these groups is affected by the $\mathrm{pH}$ value, which can alter either the internal network structure of the hydrogels or the affinity/hydrophobicity of the molecular chains, thereby altering the water absorption capacity of the gel [85]. A novel pH-sensitive hydrogel with excellent mechanical strength was prepared using oligomonomers of poly(ethylene glycol) methyl ether methacrylate (PEGMA) and poly(acrylic acid) (PAA) [86]. When immersed in solutions with a $\mathrm{pH}$ below $\sim 4$, the hydrogels exhibited a low swelling ratio with a compression strength of $\sim 8 \mathrm{MPa}$, while in solutions with a $\mathrm{pH}>4$, the hydrogels were transparent and exhibited a high swelling ratio with a compression strength of $\sim 1 \mathrm{MPa}$. The robust nature of these hydrogels over a wide $\mathrm{pH}$ range may be useful for applications such as artificial muscles and controlled release devices.

Moreover, dual or multiple smart hydrogels may be prepared by combining two or more responsive hydrogel types using interpenetrating network connection or a graft 


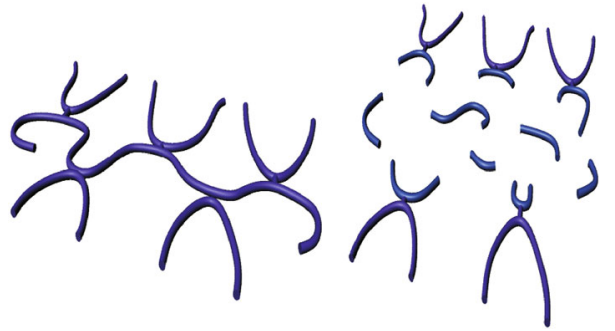

(a) General chemistry hydrogel

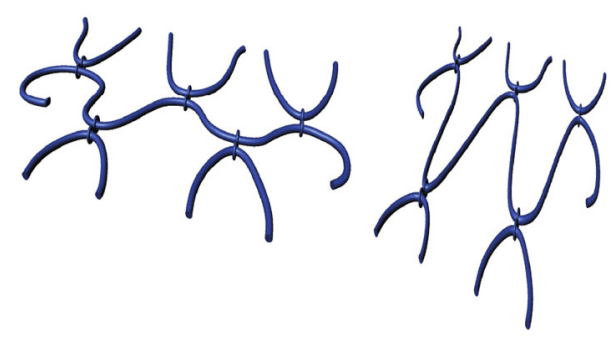

(b) Sliding hydrogel

Figure 3: General chemistry hydrogel and sliding hydrogel.

copolymerization method, among other approaches. For example, a new kind of multiple stimulus-responsive organic/inorganic hybrid hydrogel was successfully fabricated by combining a dual stimuli-responsive poly(2-(2-methoxyethoxy)ethyl methacrylate-co-oligo(ethylene glycol)methacrylate-co-acrylic acid) (PMOA) hydrogel with magnetic attapulgite $/ \mathrm{Fe}_{3} \mathrm{O}_{4}\left(\mathrm{AT}-\mathrm{Fe}_{3} \mathrm{O}_{4}\right)$ nanoparticles [87]. The resulting $\mathrm{AT}-\mathrm{Fe}_{3} \mathrm{O}_{4} / \mathrm{PMOA}$ hydrogels presented temperature/pH sensitivity, good mechanical properties, and magnetic functionality, allowing them to continue to swell under an alternating magnetic field following equilibrium swelling in deionized water [87].

2.5. Nanocomposite Hydrogels. The nanoscale dispersion of different materials in the polymeric matrix form nanocomposite hydrogels, with particle sizes ranging from 1 to $1000 \mathrm{~nm}$, and the molecular chain structure consists of branched polymers and cross-linked network polymers [21]. This uniform dispersion of inorganic components can greatly improve the strength of hydrogels [19]. Nanocomposite hydrogels are also known as hybrid hydrogels, which are formed by physical or chemical covalent cross-linking of the polymer network with raw material particles, including carbon-based nanoparticles, polymeric nanoparticles, inorganic/ceramic nanoparticles, or metal/metal oxides [19, 21, 88-90].

Yadollahi et al. reported the synthesis of carboxymethyl cellulose/ZnO nanocomposite hydrogels by in situ formation of $\mathrm{ZnO}$ nanoparticles within swollen carboxymethyl cellulose hydrogels [91]. The resulting nanocomposite hydrogels exhibited both $\mathrm{pH}$ - and salt-sensitive swelling behaviors. In addition, they exhibited increased swelling in different aqueous solutions compared with neat hydrogels. The presence of the inorganic salt can weaken hydrogen bonding between the polymer chains and water, resulting in a localized dehydration of the polyphosphate ester and thus increasing aggregation. Based on this approach, a novel biodegradable nanoscopic hydrogel was synthesized by photocross-linking salt-induced polymer assemblies [92]. This method was based on the integration of block copolymers containing polyphosphoester (poly(ethyl ethylene phosphate) (PEEP)), which could undergo a salt-induced hydrophobic-to-hydrophilic transition. A triblock copolymer of poly(ethylene glycol) (PEG) combined with PEEP was synthesized, and its end groups were then functionalized with acryloyl chloride to produce an acrylate block copolymer (Acr-PEEP-PEG-PEEPAcr). Subsequently, a diblock copolymer of PEG and PEEP
(Acr-PEEP-PEG-Lac) containing heteroacrylate and lactosyl end functional groups was also synthesized to incorporate targeting moieties into the nanogel. These block copolymers were found to be soluble in water but self-assembled into core-shell structural nanoparticles following the addition of salt. The resulting nanoparticles could become totally hydrophilic after UV cross-linking to anchor the structure and dialysis to remove salt, generating nanogel particles with an inner reservoir for water-soluble drugs. This synthesis method is both facile and biocompatible, which could waive the inconvenient purification requirements typically required following nanogel generation [92].

2.6. Sliding Hydrogels. Sliding hydrogels contain topologically interlocked noncovalent cross-links that can slide along a threaded polymer backbone [93]. A novel sliding hydrogel, pseudopolyrotaxanes of monothiolated beta-cyclodextrin threaded on poly(allyl glycidyl ether)-block-poly(ethylene glycol)-block-poly(allyl glycidyl ether), was previously prepared in water by sonication and subsequently photocrosslinked by UV irradiation, resulting in a sliding hydrogel with elasticity comparable to other hydrogels, increased stretchability, and tunable degradability under acidic conditions [16]. The benefit of these hydrogels lies in their more stable mechanical properties, as common hydrogels exhibit greater volatility under a smaller strain range. Research suggests that the mechanical strength of sliding hydrogels can adapt to the strength of mammal skin, blood vessels, and tissues; therefore, the sliding hydrogels could be used as a substitute for a variety of biological materials. Additionally, the stimulation sensitivity of sliding hydrogels may be improved by modifying cyclodextrin rings with the addition of sensitive groups $[94,95]$. For example, a novel photosensitive sliding hydrogel was prepared by adding azobenzene units to the mobile $\alpha$-cyclodextrin units of a poly(ethylene oxide)-based polyrotaxane and its photoresponsive behavior was attributed to the dynamic nature of the cross-linkers [96].

In summary, there are three primary factors that determine the performance of sliding hydrogels: the number of rings on each polymer chain, cross-linking density, and swelling properties of the hydrogels in solvents [97]. In conventional gels, the chain length between cross-linking points is uneven and the shorter chains may break more readily due to unequal chain tension under external force (Figure 3(a)). Conversely, in sliding gels, the chain length between crosslinking points is relatively uniform due to free slide of 


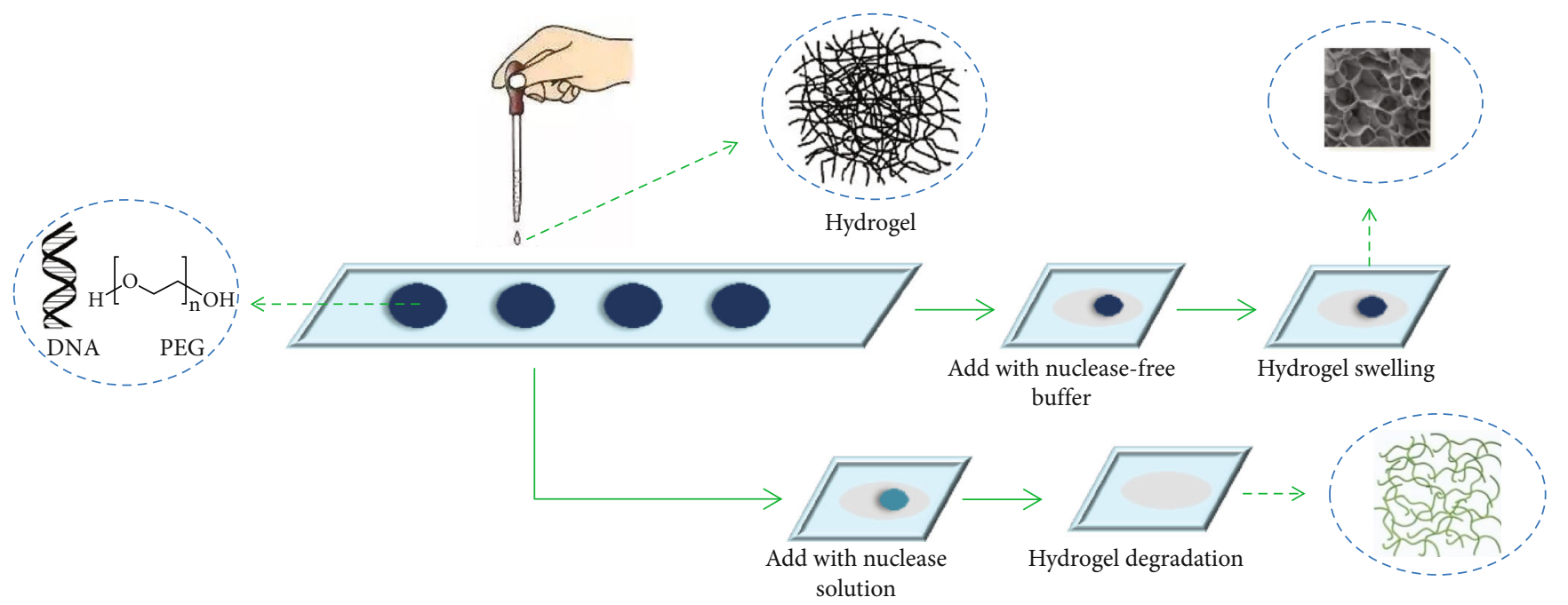

Figure 4: Hydrogel network formation via copper-free click chemistry, followed by hydrogel swelling when immersed in nuclease-free buffer or biodegradation when incubated in the presence of nucleases.

cross-linked rings under external force, so these hydrogels exhibit higher mechanical strength than conventional gels (Figure 3(b)).

2.7. Other Novel Hydrogels. In addition to the five biomedical hydrogels cited above, research has led to the development of a number of other novel hydrogels, including DNAenabled hydrogels, and magnetic hyaluronate hydrogels [98, 99]. DNA-enabled hydrogels have been synthesized through the reaction of dibenzocyclooctyne-functionalized multiarm poly(ethylene glycol) with azide-functionalized single-stranded DNA in aqueous solutions by copper-free click chemistry (Figure 4) [98]. Besides, the hydrogel contained with the adipose-derived stem cells has been reported that can augment diabetic wound healing [100]. These gels can be degraded by nucleases and may be modified for use in a variety of applications, such as drug delivery and wound healing systems. These research efforts will continue to drive the development of novel hydrogels with increased sensitivity to the area of changing environmental conditions, improving strength, elasticity, and capability of novel hydrogels; all of which can further enhance their critical roles in biomedicine.

\section{Applications of Hydrogels}

3.1. Applications in Drug Delivery Systems. In recent years, drug delivery systems capable of controlled dosage delivery for extended periods in the affected area have been vigorously developed all over the world [101]. An effective drug delivery system has three critical requirements of the structure: a region for drug storage, a controlled release rate, and a release drive [102]. Hydrogels exhibit these three functions. Moreover, hydrogels can mask the bitter taste and odor of pharmaceuticals. Thus, hydrogels have a great potential for application via oral, nasal, buccal, rectal, vaginal, eye, injection, and other administration routes. When the hydrogel is injected or transplanted into an organism, it can maintain the effective and controlled release of an embedded drug into body fluids [103]. The therapeutic effects of many lipophilic drugs are limited due to a variety of problems including poor solubility, poor dispersion, lack of uniformity, poor dissolution, low bioavailability, and lack of in vivo stability. However, when these drugs are uploaded to a hydrogel system, the above defects can be improved to some extent, resulting in solubilization, sustained release or controlled release effects, and enhanced stability and bioactivity. Conversely, small molecule drugs that are highly soluble exhibit more advantages, including improved absorption and high bioavailability, but these properties are incompatible with sustained drug delivery effects. To exploit these more desirable properties, a novel interpenetrating polymer network was synthesized through the modification of silicone elastomers with a poly(2-hydroxyethyl methacrylate) (PHEMA)-based hydrogel characterized by a surface-connected hydrophilic carrier network inside the silicone [104]. These structures were then loaded with the antibiotic ciprofloxacin, and the resulting drug release inhibited bacterial growth when placed on agar, suggesting that these hydrogels have potential for future applications in drug-releasing medical devices [104]. Additionally, floating hydrogels synthesized from kappa carrageenan containing either $\mathrm{CaCO}_{3}$ or $\mathrm{NaHCO}_{3}$ as pore forming agents have been evaluated with amoxicillin trihydrate as a model drug [105]. The hydrogels incorporating $\mathrm{CaCO}_{3}$ exhibited higher drug entrapment efficiency and longer sustained drug release than $\mathrm{NaHCO}_{3}$, indicating that $\mathrm{CaCO}_{3}$ is a viable pore-forming agent for the development of an effective floating drug delivery system [105]. Interestingly, because of the distinctive conductivity of the conducting hydrogel, they are also electrostimulated drug release devices and these devices have great advantages in lowvoltage drive and high load capacity [106]. Based on the conductive polymer poly(3-methoxydiphenylamine) and pectin hydrogels, Mongkolkitikul et al. synthesize a drug delivery system to transport ibuprofen. And the study showed that under applied electric potential, the diffusion coefficient of a 
drug is much higher than those without electric potential due to the mesh size expansion [107].

Hydrogels can also be used as a carrier for biological macromolecular drugs, mainly due to the controlled release behavior of protein drugs in polymer systems. For example, hydrophilic and hydrolytically degradable poly(ethylene glycol) (PEG) hydrogels were prepared via Michael-type addition, which were employed for sustained delivery of a monoclonal antibody against the protective antigen of anthrax. Burst release of the antibody from the matrix was avoided due to the PEG-induced precipitation. These hydrogels were able to release active antibodies in a controlled manner, for up to 56 days in vitro, by varying the polymer architectures and molecular weights of the precursors. Analyses of the secondary and tertiary protein structures and the in vitro activity of the released antibody indicated that the encapsulation and release of the drug did not affect the protein conformation or functionality, which suggested that this is a promising approach for developing PEG-based carriers capable of sustained release of therapeutic antibodies against toxins in various applications [108]. Similarly, novel discshaped hydrogel nanoparticles have been prepared by fragmentation of stearoyl macrogol-32 glycerides (Gelucire 50/13) hydrogels, and the resulting nanoparticles exhibited good physical stability due to their outer coating of PEG [109]. Moreover, these nanoparticles exhibited good loading capacity for hydrophilic macromolecules (such as lysozyme) mainly via surface adsorption, indicating their potential as effective nanocarriers for drug delivery (Figure 5).

In addition, several studies have reported the use of hydrogels as carriers for polysaccharide substances and genes. A novel high-strength photosensitive hydrogel was formed by the photoinitiated copolymerization of hydrophilic hydrogen bonding monomer (acrylamide (AAm)), hydrophobic hydrogen bonding monomer (2-vinyl-4,6diamino-1,3,5-triazine (VDT)), and a spiropyran-containing monomer, in the presence of the cross-linker poly(ethylene glycol) diacrylate. Reverse gene transfection was then successfully accomplished by anchoring the PVDT/pDNA complex nanoparticles on the gel surface through hydrogen bonding between diaminotriazine motifs prior to cell seeding [110]. Interestingly, the gene transfection level could be further increased by fibronectin modification combined with the supplementation of PVDT/pDNA complex nanoparticles after the first cycle of reverse gene transfection (i.e., sandwich gene transfection) [110].

3.2. Applications in Wound Dressings. Hydrogel materials have been used directly in contact with human tissues, absorbing exudate to form a gel, which effectively prevents the loss of body fluids and is not subject to adhesion on the wound after absorption of exudate [111]. Hydrogels can also deliver oxygen to the wound to accelerate the growth of epithelial cells and proliferation of new capillaries $[112,113]$ and can protect the wound from bacterial violations, inhibiting bacterial growth and thus promoting wound healing in general [114]. There is an unmet clinical need for wound dressings, and currently, hydrogel materials for wound dressings have entered the commercial market. These hydrogel materials can be made of spray, emulsion, or paste, with embedded anti-inflammatory drugs that can be slowly released through the gel to the injured area, which can accelerate wound healing. A class of "smart" peptide hydrogels was prepared by self-assembling of ultrashort aliphatic peptides into helical fibers, and these nanofibrous hydrogels reportedly accelerated wound closure in a rat model for partial thickness burns [115]. The regenerative properties could be further enhanced by incorporation of bioactive moieties such as growth factors and cytokines. Singh et al. reported the development of a novel hydrogel combining silver nanoparticles and polyvinyl pyrrolidone (PVP) blended with carrageenan via gamma irradiation, which could be used as wound dressings to control infection and facilitate the healing process for burns and other skin injuries [116].

3.3. Applications in Biosensors. The biosensor is a fast, accurate, and real-time detection means. Biomolecules generally are fixed either on the surface or the interior of hydrogels, connecting to the physical elements of the biosensors. The hydrogel film is the hub connecting the biomolecules and physical components. Hydrogels prepared for sensors are typically comprised of alginate, alginic acid in complex with chitosan, acrylamide, or $\mathrm{N}$-isopropyl acrylamide [117-121]. A nonenzymatic electrochemical $\mathrm{H}_{2} \mathrm{O}_{2}$ sensor was prepared by in situ fabrication of biocompatible chitosan hydrogels containing a specific recognition molecule for $\mathrm{H}_{2} \mathrm{O}_{2}$, and this sensor exhibited a fast amperometric response to $\mathrm{H}_{2} \mathrm{O}_{2}$ within $7 \mathrm{~s}$. The remarkable analytical performance of the nonenzymatic electrochemical sensor represents a promising model for durable monitoring of $\mathrm{H}_{2} \mathrm{O}_{2}$ in rat brain microdialysates, which will improve our ability to understand the biological effects of $\mathrm{H}_{2} \mathrm{O}_{2}$ on pathological and physiological processes [122]. Similarly, Devadhasan and Kim introduced a new method to quantify various $\mathrm{pH}$ solutions with a complementary metal oxide semiconductor image sensor, which produced high-accuracy analyses based on $\mathrm{pH}$ measurement [123]. In this approach, a thin film was fabricated by merging a $\mathrm{pH}$ indicator with the hydrogel matrix and the modified gel exhibited color change development across the full spectrum of $\mathrm{pH}$ ( $\mathrm{pH} 1-14)$. The complementary metal oxide semiconductor image sensor then absorbed the color intensity of the hydrogel film, and the hue value was converted into digital data with the help of an analog-to-digital converter to determine the $\mathrm{pH}$ ranges of solutions [123]. This gel may be useful for in situ $\mathrm{pH}$ sensing in the presence of toxic chemicals and chemical vapors.

3.4. Applications in Contact Lenses. Contact lenses are delicate ophthalmic medical tools for correcting vision or changing eye color for aesthetic effects, and their permeability and biocompatibility are key properties to be considered during design. Hydrogel contact lenses must be comfortable to wear, have good oxygen permeability, and potentially have the capacity to assist in the treatment of eye diseases, which is the reason why hydrogels represent important manufacturing materials for contact lenses [124]. The majority of soft contact lenses are comprised of poly(2hydroxyethyl methacrylate) hydrogels cross-linked with 


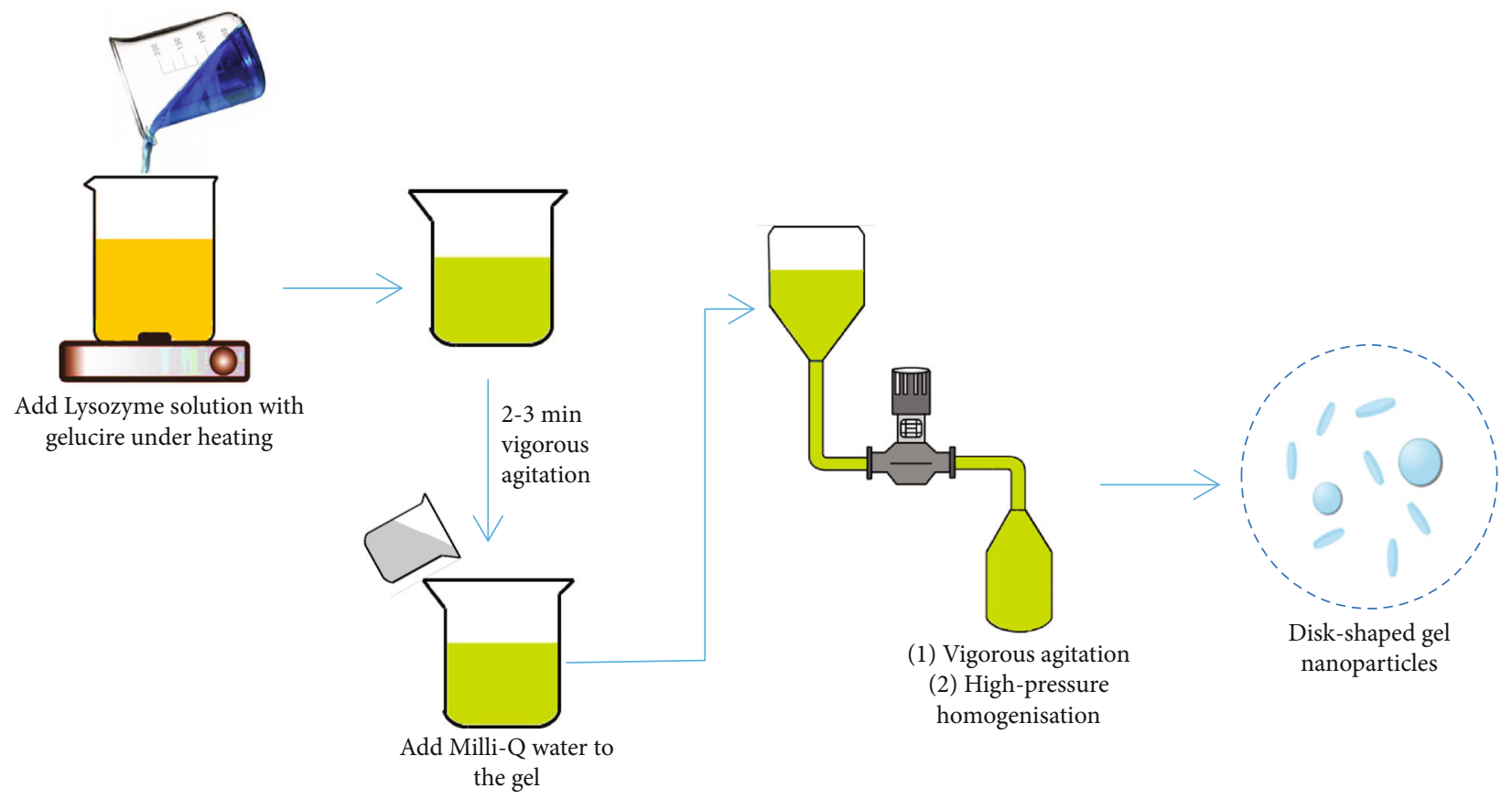

FIGURE 5: Schematic illustration of the fabrication process of lysozyme-loaded disc-shaped gel nanoparticles.

ethylene glycol dimethacrylate or silicone [125]. Poly(2hydroxyethyl methacrylate) hydrogels have several advantages: relatively high water content, thermal and chemical stability, tunable mechanical properties, and oxygen permeability, which are very important for safe daily wear [126]. Poly(2-hydroxyethyl methacrylate) (p(HEMA)) soft contact lenses have been prepared by thermal or photopolymerization of HEMA solutions containing ethylene glycol dimethacrylate as the cross-linker and different proportions of $\mathrm{N}$-vinyl-2-pyrrolidone or methacrylic acid as comonomers [127]. The drug loading capacity and release properties of p(HEMA)-based soft contact lenses were improved based on the optimization of the hydrogel composition and microstructural modifications using water during the polymerization process [127].

3.5. Applications in Tissue Engineering. The objective of tissue engineering research and development is to produce biological alternatives to restore, maintain, or improve the morphology and function of damaged tissues and organs, thereby achieving reconstruction by applying principles and methods from both cell biology and engineering [128]. Tissue engineering has also been defined as "regenerative medicine." Specifically, donor cells, after amplification in vitro, are seeded for growth onto a biodegradable three-dimensional scaffold. The resulting complex can then be implanted into the body or at targeted sites, where the implanted cells continue to proliferate and secrete an extracellular matrix [129]. As the scaffold degrades, new tissues or organs will form bearing the same shape and function as the damaged tissues or organs. Tissue engineering represents an extraordinarily important, emerging area of research with enormous scientific value and broad application prospects and will be a major focus of life science researches in the 21 st century.
Organ transplantation will be replaced by the development and manufacturing of synthetic tissues and organs. One of the key technologies of tissue engineering is the preparation of a biocompatible and biodegradable cell scaffold, and hydrogels represent a large class of materials that can function as tissue engineering scaffolds [130]. Hydrogels are a type of three-dimensional scaffolds with chemical or physical cross-linking structures, which can absorb and retain large amounts of water, yet remain insoluble in water. Hydrogels have been widely used as scaffold materials in tissue engineering for several reasons. First, hydrogels are soft and flexible, similar to soft tissues in vivo. Second, hydrogels in the liquid state can be implanted in the body by injection, where they can quickly fill tissue defects by forming irregular nonflowing semisolids [52]. This method is simple and also circumvents risks associated with trauma, infection, and scar formation by surgical implantation. Third, the threedimensional network structure of a hydrogel is similar to a natural extracellular matrix, which will eventually promote cell engraftment, adhesion, and growth by adjusting the porosity and pore size and increasing the internal surface area [131]. Fourth, hydrogels are rich in water (up to 99\%), which is beneficial for the transportation of oxygen, nutrients, and cellular metabolites, in addition to reducing friction and mechanical stimulation of the surrounding tissues. Additionally, the inclusion of cells prior to gelation results in a more uniform distribution of cells throughout the scaffold, thus increasing plating efficiency [132].

A variety of potential medical hydrogels have been investigated in recent years, such as poly(lactic-co-glycolic acid) scaffolds and polylactic acid scaffolds combined with osteoblasts in bone tissue engineering, filamentous collagen materials in neural tissue engineering, and cellulose acetate scaffolds combined with chondrocytes in cartilage tissue 
engineering [133-135]. Vo et al. reported the osteogenic potential of injectable, dual thermally and chemically gelable composite hydrogels for mesenchymal stem cell delivery in vitro and in vivo [136]. A novel composite hydrogel containing copolymer macromers of $\mathrm{N}$-isopropylacrylamide was prepared by the incorporation of gelatin microparticles as enzymatically digestible porogens and sites for cellular attachment. Results indicated that these injectable, dualgelling cell-laden composite hydrogels could facilitate bone ingrowth and integration, ensuring further research for bone tissue engineering [136]. Also, a novel injectable hydrogel based on a glycopolypeptide was prepared by an enzymatic cross-linking reaction in the presence of horseradish peroxidase and hydrogen peroxide, in which the glycopolypeptide was synthesized through conjugation of poly( $\gamma$-propargyl-L-glutamate) with azido-modified mannose and 3-(4-hydroxyphenyl) propanamide via click chemistry [137]. The resulting hydrogels displayed good cytocompatibility in vitro and were rapidly formed in situ after subcutaneous injection into rats, exhibiting acceptable biocompatibility desirable biodegradation in vivo. Interestingly, the glycopolypeptide hydrogels containing chondrocytes in the subcutaneous model of nude mice were observed to maintain the chondrocyte phenotype and produce the cartilaginous specific matrix, indicating that the biomimetic glycopolypeptide-based hydrogels represent potential three-dimensional scaffolds for cartilage tissue engineering [137]. Due to additional benefit in electrical conductivity, conductive hydrogels play the tremendous role in tissue engineering. Homogenously electrical double network based on conducting polymer poly(3-thiophene acetic acid) (PTAA) has been reported to be used in myocardial tissue engineering. It can support BADSC adhesion, and reduce inflammation in vivo, and the PTAA in this hydrogel can significantly enhance the differentiation potency of BADSCs to cardiomyocytes, increase the expression of myocardial specific proteins cTnT and $\alpha$-actinin, promote intercellular communication ability, and increase the expression of connexin 43. More importantly, electrical stimulation can enhance the effect of PTAA [138].

\section{Conclusions and Future Perspectives}

Hydrogels are new functional polymer materials experiencing rapid development. New biomedical hydrogels have been observed to exhibit improved degradation and mechanical properties, thereby overcoming deficiencies found in traditional hydrogels and expanding the potential roles of hydrogels in the field of biomedical applications. However, for applications based on particular tissues or organs, much research remains to develop hydrogels capable of functioning as replacements for real tissues. Future studies into biomedical hydrogels will be needed to address the following: (i) the swelling rate of hydrogels should be controlled while improving their mechanical properties, meeting the size requirements of tissues and organs; (ii) their biocompatibility should be enhanced to achieve simulation of the extracellular matrix structure and functions; (iii) the degradation rate of hydrogels should be controllable, conforming to tissue- specific mechanical properties and regeneration needs; and (iv) hydrogels should be combined with other materials to achieve the complex structural and functional components necessary to act as replacements for specific organs. In summary, while technical problems associated with the synthesis and application of hydrogel materials remain to be resolved, it is clear that continuing research will eventually overcome these problems, leading to a revolutionary new model for bioengineering and advances in tissue replacement and regeneration.

\section{Conflicts of Interest}

The authors declare that there is no conflict of interest regarding the publication of this paper.

\section{Authors' Contributions}

Ke Wang and Yuting Hao contributed equally to this work.

\section{References}

[1] A. S. Hoffman, "Hydrogels for biomedical applications," Advanced drug delivery reviews., vol. 64, pp. 18-23, 2012.

[2] E. M. Ahmed, "Hydrogel: Preparation, characterization, and applications: A review," Journal of Advanced Research, vol. 6, no. 2, pp. 105-121, 2015.

[3] H. Hosseini, H. Tenhu, and S. Hietala, "Rheological properties of thermoresponsive nanocomposite hydrogels," Journal of Applied Polymer Science, vol. 133, no. 11, 2016.

[4] C. Chang and L. Zhang, "Cellulose-based hydrogels: present status and application prospects," Carbohydrate polymers., vol. 84, no. 1, pp. 40-53, 2011.

[5] K. Nielsen, H. Vorum, N. Ehlers, N. Aagaard, J. Hjortdal, and B. Honoré, "Tear film proteins deposited on high water content contact lenses identified with two-dimensional gel electrophoresis and mass spectrometry," Acta ophthalmologica., vol. 93, no. 7, pp. 658-662, 2015.

[6] E. A. Kamoun, X. Chen, M. S. Mohy Eldin, and E.-R. S. Kenawy, "Crosslinked poly (vinyl alcohol) hydrogels for wound dressing applications: a review of remarkably blended polymers," Arabian Journal of Chemistry., vol. 8, no. 1, pp. 1-14, 2015.

[7] C. Yao, Z. Liu, C. Yang et al., "Smart Hydrogels: Poly(Nisopropylacrylamide)-Clay Nanocomposite Hydrogels with Responsive Bending Property as Temperature-Controlled Manipulators (Adv. Funct. Mater. 20/2015)," Advanced Functional Materials., vol. 25, no. 20, p. 3104, 2015.

[8] N. Rodkate, B. Rutnakornpituk, U. Wichai, G. Ross, and M. Rutnakornpituk, "Smart carboxymethylchitosan hydrogels that have thermo-and $\mathrm{pH}$-responsive properties," Journal of Applied Polymer Science, vol. 132, no. 8, 2015.

[9] A. Tudor, L. Florea, S. Gallagher, J. Burns, and D. Diamond, "Poly (ionic liquid) semi-interpenetrating network multiresponsive hydrogels," Sensors, vol. 16, no. 2, p. 219, 2016.

[10] M. Karimi, P. S. Zangabad, A. Ghasemi, and M. R. Hamblin, "Electrical and mechanical-responsive nanocarriers," in Smart External Stimulus-Responsive Nanocarriers for Drug and Gene Delivery, 2015.

[11] M. K. Lima-Tenório, E. T. Tenório-Neto, F. P. Garcia et al., "Hydrogel nanocomposite based on starch and Co-doped 
zinc ferrite nanoparticles that shows magnetic fieldresponsive drug release changes," Journal of Molecular Liquids., vol. 210, pp. 100-105, 2015.

[12] B. V. Sridhar, J. L. Brock, J. S. Silver, J. L. Leight, M. A. Randolph, and K. S. Anseth, "Tissue engineering: development of a cellularly degradable PEG hydrogel to promote articular cartilage extracellular matrix deposition (Adv. Healthcare Mater. 5/2015)," Advanced healthcare materials, vol. 4, no. 5, p. 635, 2015.

[13] L. Gritsch, F. L. Motta, and S. Fare, "History and applications of hydrogels," Journal of Biomedical Science, vol. 04, no. 02, p. 13, 2015.

[14] M. Alhede, L. H. Christensen, and T. Bjarnsholt, "Microbial biofilms and adverse reactions to gel fillers used in cosmetic surgery," in Biofilm-based Healthcare-associated Infections, pp. 45-52, Springer, Cham, 2015.

[15] A. Blumlein and J. J. McManus, "Bigels formed via spinodal decomposition of unfolded protein," Journal of Materials Chemistry B., vol. 3, no. 17, pp. 3429-3435, 2015.

[16] T. Murakami, B. V. K. J. Schmidt, H. R. Brown, and C. J. Hawker, "One-pot "click" fabrication of slide-ring gels," Macromolecules, vol. 48, no. 21, pp. 7774-7781, 2015.

[17] L. Cai, R. E. Dewi, and S. C. Heilshorn, "Injectable hydrogels with in situ double network formation enhance retention of transplanted stem cells," Advanced Functional Materials., vol. 25, no. 9, pp. 1344-1351, 2015.

[18] S. Noori, M. Kokabi, and Z. M. Hassan, "Nanoclay enhanced the mechanical properties of poly (vinyl alcohol)/chitosan/montmorillonite nanocomposite hydrogel as wound dressing," Procedia Materials Science, vol. 11, pp. 152-156, 2015.

[19] S. Merino, C. Martín, K. Kostarelos, M. Prato, and E. Vázquez, "Nanocomposite hydrogels: 3D polymer-nanoparticle synergies for on-demand drug delivery," ACS Nano, vol. 9, no. 5, pp. 4686-4697, 2015.

[20] Q. Li, D. G. Barrett, P. B. Messersmith, and N. HoltenAndersen, "Controlling Hydrogel MechanicsviaBioInspired Polymer-Nanoparticle Bond Dynamics," ACS nano, vol. 10, no. 1, pp. 1317-1324, 2015.

[21] A. K. Gaharwar, N. A. Peppas, and A. Khademhosseini, "Nanocomposite hydrogels for biomedical applications," Biotechnology and Bioengineering, vol. 111, no. 3, pp. 441-453, 2014.

[22] R. Balint, N. J. Cassidy, and S. H. Cartmell, "Conductive polymers: towards a smart biomaterial for tissue engineering," Acta Biomaterialia, vol. 10, no. 6, pp. 2341-2353, 2014.

[23] A. M. Kloxin, C. J. Kloxin, C. N. Bowman, and K. S. Anseth, "Mechanical properties of cellularly responsive hydrogels and their experimental determination," Advanced Materials, vol. 22, no. 31, pp. 3484-3494, 2010.

[24] K. Gilmore, A. J. Hodgson, B. Luan, C. J. Small, and G. G. Wallace, "Preparation of hydrogel/conducting polymer composites," Polymer Gels and Networks, vol. 2, no. 2, pp. 135-143, 1994.

[25] K. Sharma, V. Kumar, B. S. Kaith, S. Kalia, and H. C. Swart, "Conducting polymer hydrogels and their applications," in Conducting Polymer Hybrids, pp. 193-221, Springer, Cham, 2017.

[26] Q. Tang, J. Lin, J. Wu, C. Zhang, and S. Hao, "Two-steps synthesis of a poly(acrylate-aniline) conducting hydrogel with an interpenetrated networks structure," Carbohydrate Polymers., vol. 67 , no. 3, pp. 332-336, 2007.
[27] Q. Tang, J. Wu, and J. Lin, "A multifunctional hydrogel with high conductivity, $\mathrm{pH}$-responsive, thermo-responsive and release properties from polyacrylate/polyaniline hybrid," Carbohydrate Polymers., vol. 73, no. 2, pp. 315-321, 2008.

[28] J. Lin, Q. Tang, D. Hu, X. Sun, Q. Li, and J. Wu, "Electric field sensitivity of conducting hydrogels with interpenetrating polymer network structure," Colloids and Surfaces A: Physicochemical and Engineering Aspects, vol. 346, no. 1-3, pp. 177183, 2009.

[29] Q. Tang, J. Wu, H. Sun, S. Fan, D. Hu, and J. Lin, "Superabsorbent conducting hydrogel from poly(acrylamide-aniline) with thermo-sensitivity and release properties," Carbohydrate Polymers., vol. 73, no. 3, pp. 473-481, 2008.

[30] L. Zhang, W. Du, A. Nautiyal, Z. Liu, and X. Zhang, "Recent progress on nanostructured conducting polymers and composites: synthesis, application and future aspects," Science China Materials, vol. 61, no. 3, pp. 303-352, 2018.

[31] Z. Liu, Y. Liu, L. Zhang et al., "Controlled synthesis of transition metal/conducting polymer nanocomposites," Nanotechnology, vol. 23, no. 33, p. 335603, 2012.

[32] L. Ghasemi-Mobarakeh, M. P. Prabhakaran, M. Morshed et al., "Application of conductive polymers, scaffolds and electrical stimulation for nerve tissue engineering," Journal of Tissue Engineering and Regenerative Medicine, vol. 5, no. 4, pp. e17-e35, 2011.

[33] K. M. Sajesh, R. Jayakumar, S. V. Nair, and K. P. Chennazhi, "Biocompatible conducting chitosan/polypyrrole-alginate composite scaffold for bone tissue engineering," International journal of biological macromolecules., vol. 62, pp. 465-471, 2013.

[34] C. Shi, Y. He, M. Ding, Y. Wang, and J. Zhong, "Nanoimaging of food proteins by atomic force microscopy. Part I: components, imaging modes, observation ways, and research types," Trends in Food Science \& Technology, vol. 87, pp. 3-13, 2019.

[35] C. Shi, Y. He, M. Ding, Y. Wang, and J. Zhong, "Nanoimaging of food proteins by atomic force microscopy. Part II: application for food proteins from different sources," Trends in Food Science \& Technology, vol. 87, pp. 14-25, 2019.

[36] F. García, J. Buendía, S. Ghosh, A. Ajayaghosh, and L. Sánchez, "Luminescent and conductive supramolecular polymers obtained from an $\mathrm{N}$-annulated perylenedicarboxamide," Chemical Communications, vol. 49, no. 81, pp. 92789280, 2013.

[37] D. Mawad, E. Stewart, D. L. Officer et al., "Conducting polymer hydrogels: a single component conducting polymer hydrogel as a scaffold for tissue engineering (Adv. Funct. Mater. 13/2012)," Advanced Functional Materials., vol. 22, no. 13, pp. 2691-2691, 2012.

[38] G. Kaur, R. Adhikari, P. Cass, M. Bown, and P. Gunatillake, "Electrically conductive polymers and composites for biomedical applications," Rsc Advances., vol. 5, no. 47, pp. 37553-37567, 2015.

[39] Y. Shi, J. Zhang, A. M. Bruck et al., "A tunable 3D nanostructured conductive gel framework electrode for highperformance lithium ion batteries," Advanced Materials, vol. 29, no. 22, p. 1603922, 2017.

[40] G. Cai, J. Wang, K. Qian, J. Chen, S. Li, and P. S. Lee, "Extremely Stretchable Strain Sensors Based on Conductive Self-Healing Dynamic Cross-Links Hydrogels for HumanMotion Detection," Advanced Science, vol. 4, no. 2, p. 1600190, 2017. 
[41] Q. Rong, W. Lei, and M. Liu, "Conductive hydrogels as smart materials for flexible electronic devices," ChemistryA European Journal., vol. 24, no. 64, pp. 16930-16943, 2018.

[42] W. Xu, Y. Ding, Y. Yu, S. Jiang, L. Chen, and H. Hou, "Highly foldablePANi@CNTs/PU dielectric composites toward thinfilm capacitor application," Materials Letters., vol. 192, pp. 25-28, 2017.

[43] J. Han, K. Lu, Y. Yue et al., "Nanocellulose-templated assembly of polyaniline in natural rubber-based hybrid elastomers toward flexible electronic conductors," Industrial Crops and Products, vol. 128, pp. 94-107, 2019.

[44] S. J. Devaki, R. K. Narayanan, and S. Sarojam, "Electrically conducting silver nanoparticle-polyacrylic acid hydrogel by in situ reduction and polymerization approach," Materials Letters., vol. 116, no. 2, pp. 135-138, 2014.

[45] Y. Xiang and D. Chen, "Preparation of a novel pH-responsive silver nanoparticle/poly (HEMA-PEGMA-MAA) composite hydrogel," European Polymer Journal., vol. 43, no. 10, pp. 4178-4187, 2007.

[46] L. Pan, G. Yu, D. Zhai et al., "Hierarchical nanostructured conducting polymer hydrogel with high electrochemical activity," Proceedings of the National Academy of Sciences of the United States of America., vol. 109, no. 24, pp. 92879292, 2012.

[47] N. Park, S. C. Chae, I. T. Kim, and J. Hur, "Fabrication of selfhealable and patternable polypyrrole/agarose hybrid hydrogels for smart bioelectrodes," Journal of Nanoscience and Nanotechnology, vol. 16, no. 2, pp. 1400-1404, 2016.

[48] J. Hur, K. Im, S. W. Kim et al., "Polypyrrole/agarose-based electronically conductive and reversibly restorable hydrogel," ACS Nano, vol. 8, no. 10, pp. 10066-10076, 2014.

[49] S. Zhou, M. Wang, X. Chen, and F. Xu, "Facile template synthesis of microfibrillated cellulose/polypyrrole/silver nanoparticles hybrid aerogels with electrical conductive and pressure responsive properties," Acs Sustainable Chemistry \& Engineering., vol. 3, no. 12, pp. 3346-3354, 2015.

[50] S. S. Sivan, S. Roberts, J. P. G. Urban et al., "Injectable hydrogels with high fixed charge density and swelling pressure for nucleus pulposus repair: Biomimetic glycosaminoglycan analogues," Acta Biomaterialia, vol. 10, no. 3, pp. 1124-1133, 2014.

[51] J.-A. Yang, J. Yeom, B. W. Hwang, A. S. Hoffman, and S. K. Hahn, "In situ-forming injectable hydrogels for regenerative medicine," Progress in Polymer Science., vol. 39, no. 12, pp. 1973-1986, 2014.

[52] H. Park, B. Choi, J. Hu, and M. Lee, "Injectable chitosan hyaluronic acid hydrogels for cartilage tissue engineering," Acta Biomaterialia, vol. 9, no. 1, pp. 4779-4786, 2013.

[53] J. Hu, Y. Hou, H. Park et al., "Visible light crosslinkable chitosan hydrogels for tissue engineering," Acta Biomaterialia, vol. 8, no. 5, pp. 1730-1738, 2012.

[54] S. Koutsopoulos and S. Zhang, Multi-layered injectable selfassembling peptide scaffold hydrogels for long-term sustained release of human antibodies, Google Patents, 9700521B2, Washington, DC, USA, 2014.

[55] S. Koutsopoulos and S. Zhang, "Two-layered injectable selfassembling peptide scaffold hydrogels for long-term sustained release of human antibodies," Journal of Controlled Release, vol. 160, no. 3, pp. 451-458, 2012.

[56] F. Corrente, H. M. Abu Amara, S. Pacelli, P. Paolicelli, and M. A. Casadei, "Novel injectable and in situ cross-linkable hydrogels of dextran methacrylate and scleroglucan derivatives: preparation and characterization," Carbohydrate polymers., vol. 92, no. 2, pp. 1033-1039, 2013.

[57] S. Pacelli, P. Paolicelli, I. Dreesen, S. Kobayashi, A. Vitalone, and M. A. Casadei, "Injectable and photocross-linkable gels based on gellan gum methacrylate: a new tool for biomedical application," International journal of biological macromolecules., vol. 72, pp. 1335-1342, 2015.

[58] O. Yom-Tov, L. Neufeld, D. Seliktar, and H. Bianco-Peled, “A novel design of injectable porous hydrogels with in situ pore formation," Acta Biomaterialia, vol. 10, no. 10, pp. 42364246, 2014.

[59] Q. Chen, L. Zhu, C. Zhao, Q. Wang, and J. Zheng, "A robust, one-pot synthesis of highly mechanical and recoverable double network hydrogels using thermoreversible sol-gel polysaccharide," Advanced Materials, vol. 25, no. 30, pp. 4171-4176, 2013.

[60] T. Hattori, K. Ishii, T. Tominaga, Y. Osada, and T. Tahara, "A fluorescence study on the local environment of hydrogels: double-network hydrogels having extraordinarily high mechanical strength and its constituent single-network hydrogels," Chemical Physics., vol. 419, pp. 172-177, 2013.

[61] H. Yin, T. Akasaki, T. Lin Sun et al., "Double network hydrogels from polyzwitterions: high mechanical strength and excellent anti-biofouling properties," Journal of Materials Chemistry B., vol. 1, no. 30, pp. 3685-3693, 2013.

[62] H. Shin, B. D. Olsen, and A. Khademhosseini, "The mechanical properties and cytotoxicity of cell-laden double-network hydrogels based on photocrosslinkable gelatin and gellan gum biomacromolecules," Biomaterials, vol. 33, no. 11, pp. 3143-3152, 2012.

[63] S. J. Buwalda, K. W. M. Boere, P. J. Dijkstra, J. Feijen, T. Vermonden, and W. E. Hennink, "Hydrogels in a historical perspective: from simple networks to smart materials," Journal of Controlled Release, vol. 190, pp. 254-273, 2014.

[64] T. Kataoka, Y. Ishioka, M. Mizuhata, H. Minami, and T. Maruyama, "Highly conductive ionic-liquid gels prepared with orthogonal double networks of a low-molecularweight gelator and cross-linked polymer," ACS applied materials \& interfaces., vol. 7, no. 41, pp. 23346-23352, 2015.

[65] Y. Zhang, Y. Li, and W. Liu, "Dipole-dipole and H-bonding interactions significantly enhance the multifaceted mechanical properties of thermoresponsive shape memory hydrogels," Advanced Functional Materials., vol. 25, no. 3, pp. 471-480, 2015.

[66] Y. H. Na, "Double network hydrogels with extremely high toughness and their applications," Korea-Australia Rheology Journal., vol. 25, no. 4, pp. 185-196, 2013.

[67] C. Chassenieux and C. Tsitsilianis, "Recent trends in $\mathrm{pH} /$ thermo-responsive self-assembling hydrogels: from polyions to peptide-based polymeric gelators," Soft Matter, vol. 12, no. 5, pp. 1344-1359, 2016.

[68] M. A. Haque, T. Kurokawa, and J. P. Gong, "Super tough double network hydrogels and their application as biomaterials," Polymer, vol. 53, no. 9, pp. 1805-1822, 2012.

[69] C. Chang, K. Han, and L. Zhang, "Structure and properties of cellulose/poly( $\mathrm{N}$-isopropylacrylamide) hydrogels prepared by IPN strategy," Polymers for Advanced Technologies., vol. 22, no. 9, pp. 1329-1334, 2011. 
[70] J.-Y. Sun, X. Zhao, W. R. K. Illeperuma et al., "Highly stretchable and tough hydrogels," Nature, vol. 489, no. 7414, pp. 133-136, 2012.

[71] A. Döring, W. Birnbaum, and D. Kuckling, "Responsive hydrogels-structurally and dimensionally optimized smart frameworks for applications in catalysis, micro-system technology and material science," Chemical Society Reviews., vol. 42, no. 17, pp. 7391-7420, 2013.

[72] M. C. Koetting, J. T. Peters, S. D. Steichen, and N. A. Peppas, "Stimulus-responsive hydrogels: theory, modern advances, and applications," Materials Science and Engineering: $R$ : Reports., vol. 93, pp. 1-49, 2015.

[73] X. Wang, Y. Zhang, W. Xue, H. Wang, X. Qiu, and Z. Liu, "Thermo-sensitive hydrogel PLGA-PEG-PLGA as a vaccine delivery system for intramuscular immunization," Journal of Biomaterials Applications., vol. 31, no. 6, pp. 923-932, 2016.

[74] M. Krogsgaard, M. A. Behrens, J. S. Pedersen, and H. Birkedal, "Self-healing mussel-inspired multi-pHresponsive hydrogels," Biomacromolecules, vol. 14, no. 2, pp. 297-301, 2013.

[75] A. Katchalsky, "Rapid swelling and deswelling of reversible gels of polymeric acids by ionization," Cellular and Molecular Life Sciences., vol. 5, no. 8, pp. 319-320, 1949.

[76] M. P. M. Dicker, I. P. Bond, J. M. Rossiter, C. F. J. Faul, and P. M. Weaver, "Modelling and Analysis of $\mathrm{pH}$ Responsive Hydrogels for the Development of Biomimetic Photo-Actuating Structures," MRS Proceedings, vol. 1718, pp. 65-70, 2015.

[77] C. Scherzinger, A. Schwarz, A. Bardow, K. Leonhard, and W. Richtering, "Cononsolvency of poly- $\mathrm{N}$-isopropyl acrylamide (PNIPAM): microgels versus linear chains and macrogels," Current Opinion in Colloid \& Interface Science., vol. 19, no. 2, pp. 84-94, 2014.

[78] Y. Işikver and D. Saraydin, "Environmentally sensitive hydrogels: N-isopropyl acrylamide/acrylamide/mono-, Di-, tricarboxylic acid crosslinked polymers," Polymer Engineering \& Science., vol. 55, no. 4, pp. 843-851, 2015.

[79] J. Wang, Q. Zhong, J. Wu, and T. Chen, "Thermo-responsive textiles," in Handbook of Smart Textiles, pp. 1-27, 2014.

[80] W. Wei, X. Hu, X. Qi et al., "A novel thermo-responsive hydrogel based on salecan and poly (N-isopropylacrylamide): synthesis and characterization," Colloids and Surfaces B: Biointerfaces., vol. 125, pp. 1-11, 2015.

[81] C. H. Hofmann, F. A. Plamper, C. Scherzinger, S. Hietala, and W. Richtering, "NEXT Cononsolvency Revisited: Solvent Entrapment by $N$-Isopropylacrylamide and N,N-Diethylacrylamide Microgels in Different Water/Methanol Mixtures," Macromolecules, vol. 46, no. 2, pp. 523-532, 2012.

[82] P. Mukhopadhyay, K. Sarkar, S. Bhattacharya, A. Bhattacharyya, R. Mishra, and P. P. Kundu, "pH sensitive $\mathrm{N}$-succinyl chitosan grafted polyacrylamide hydrogel for oral insulin delivery," Carbohydrate polymers., vol. 112, pp. 627-637, 2014.

[83] T. Garg, S. Singh, and A. K. Goyal, "Stimuli-sensitive hydrogels: an excellent carrier for drug and cell delivery," Critical Reviews $^{T M}$ in Therapeutic Drug Carrier Systems, vol. 30, no. 5, pp. 369-409, 2013.

[84] J. Yang, J. Chen, D. Pan, Y. Wan, and Z. Wang, "pH-sensitive interpenetrating network hydrogels based on chitosan derivatives and alginate for oral drug delivery," Carbohydrate polymers., vol. 92, no. 1, pp. 719-725, 2013.
[85] J. Liu, Y. Huang, A. Kumar et al., "pH-sensitive nano-systems for drug delivery in cancer therapy," Biotechnology advances., vol. 32, no. 4, pp. 693-710, 2014.

[86] S. Naficy, J. M. Razal, P. G. Whitten, G. G. Wallace, and G. M. Spinks, "A pH-sensitive, strong double-network hydrogel: poly (ethylene glycol) methyl ether methacrylates-poly (acrylic acid)," Journal of Polymer Science Part B: Polymer Physics., vol. 50, no. 6, pp. 423-430, 2012.

[87] Y. Wang, A. Dong, Z. Yuan, and D. Chen, "Fabrication and characterization of temperature-, $\mathrm{pH}$ - and magnetic-fieldsensitive organic/inorganic hybrid poly (ethylene glycol)based hydrogels," Colloids and Surfaces A: Physicochemical and Engineering Aspects., vol. 415, pp. 68-76, 2012.

[88] S. Roy, A. Baral, and A. Banerjee, "An amino-acid-based selfhealing hydrogel: modulation of the self-healing properties by incorporating carbon-based nanomaterials," Chemistry- $A$ European Journal., vol. 19, no. 44, pp. 14950-14957, 2013.

[89] H.-P. Cong, X.-C. Ren, P. Wang, and S.-H. Yu, "Macroscopic multifunctional graphene-based hydrogels and aerogels by a metal ion induced self-assembly process," ACS Nano, vol. 6, no. 3, pp. 2693-2703, 2012.

[90] H. Hezaveh and I. I. Muhamad, "Impact of metal oxide nanoparticles on oral release properties of $\mathrm{pH}$-sensitive hydrogel nanocomposites," International journal of biological macromolecules., vol. 50, no. 5, pp. 1334-1340, 2012.

[91] M. Yadollahi, I. Gholamali, H. Namazi, and M. Aghazadeh, "Synthesis and characterization of antibacterial carboxymethyl cellulose/ZnO nanocomposite hydrogels," International journal of biological macromolecules., vol. 74, pp. 136-141, 2015.

[92] Y.-C. Wang, J. Wu, Y. Li, J.-Z. Du, Y.-Y. Yuan, and J. Wang, "Engineering nanoscopic hydrogels via photo-crosslinking salt-induced polymer assembly for targeted drug delivery," Chemical Communications., vol. 46, no. 20, pp. 3520-3522, 2010.

[93] C. W. Peak, J. J. Wilker, and G. Schmidt, "A review on tough and sticky hydrogels," Colloid and Polymer Science., vol. 291, no. 9, pp. 2031-2047, 2013.

[94] Y. Cui, M. Tan, A. Zhu, and M. Guo, "Strain hardening and highly resilient hydrogels crosslinked by chain-extended reactive pseudo-polyrotaxane," RSC Advances., vol. 4, no. 100, pp. 56791-56797, 2014.

[95] M. Du, Y. Zhang, Y. Song, and Q. Zheng, "Negative velocity dependence of friction for poly (2-acrylamido-2-methyl propanesulfonic acid) hydrogel sliding against a glass surface in the low-velocity region," Journal of Polymer Science Part B: Polymer Physics., vol. 52, no. 11, pp. 765-772, 2014.

[96] T. Sakai, H. Murayama, S. Nagano et al., "Photoresponsive slide-ring gel," Advanced Materials, vol. 19, no. 15, pp. 2023-2025, 2007.

[97] G. Fleury, G. Schlatter, C. Brochon et al., "Topological polymer networks with sliding cross-link points: the "sliding gels". Relationship between their molecular structure and the viscoelastic as well as the swelling properties," Macromolecules, vol. 40, no. 3, pp. 535-543, 2007.

[98] K. Barker, S. K. Rastogi, J. Dominguez et al., "Biodegradable DNA-enabled poly (ethylene glycol) hydrogels prepared by copper-free click chemistry," Journal of Biomaterials Science, Polymer Edition., vol. 27, no. 1, pp. 22-39, 2016.

[99] I. Y. Tóth, G. Veress, M. Szekeres, E. Illés, and E. Tombácz, "Magnetic hyaluronate hydrogels: preparation and 
characterization," Journal of Magnetism and Magnetic Materials., vol. 380, pp. 175-180, 2015.

[100] L. Kaisang, W. Siyu, F. Lijun, P. Daoyan, C. J. Xian, and S. Jie, "Adipose-derived stem cells seeded in Pluronic F-127 hydrogel promotes diabetic wound healing," Journal of Surgical Research., vol. 217, pp. 63-74, 2017.

[101] O. Tacar, P. Sriamornsak, and C. R. Dass, "Doxorubicin: an update on anticancer molecular action, toxicity and novel drug delivery systems," Journal of Pharmacy and Pharmacology., vol. 65, no. 2, pp. 157-170, 2013.

[102] C. Gong, T. Qi, X. Wei et al., "Thermosensitive polymeric hydrogels as drug delivery systems," Current medicinal chemistry., vol. 20, no. 1, pp. 79-94, 2013.

[103] G. W. Ashley, J. Henise, R. Reid, and D. V. Santi, "Hydrogel drug delivery system with predictable and tunable drug release and degradation rates," Proceedings of the National Academy of Sciences., vol. 110, no. 6, pp. 2318-2323, 2013.

[104] S. L. Steffensen, M. H. Vestergaard, E. H. Møller et al., "Soft hydrogels interpenetrating silicone-a polymer network for drug-releasing medical devices," Journal of Biomedical Materials Research Part B: Applied Biomaterials., vol. 104, no. 2, pp. 402-410, 2016.

[105] S. Selvakumaran, I. I. Muhamad, and S. I. Abd Razak, "Evaluation of kappa carrageenan as potential carrier for floating drug delivery system: effect of pore forming agents," Carbohydrate polymers., vol. 135, pp. 207-214, 2016.

[106] L. M. Lira and S. I. Córdoba de Torresi, "Conducting polymer-hydrogel composites for electrochemical release devices: synthesis and characterization of semi-interpenetrating polyaniline-polyacrylamide networks," Electrochemistry Communications, vol. 7, no. 7, pp. 717-723, 2005.

[107] S. Mongkolkitikul, N. Paradee, and A. Sirivat, "Electrically controlled release of ibuprofen from conductive poly(3methoxydiphenylamine)/crosslinked pectin hydrogel," European Journal of Pharmaceutical Sciences., vol. 112, pp. 20-27, 2018.

[108] Y. Liang, M. V. Coffin, S. D. Manceva, J. A. Chichester, R. M. Jones, and K. L. Kiick, "Controlled release of an anthrax toxin-neutralizing antibody from hydrolytically degradable polyethylene glycol hydrogels," Journal of Biomedical Materials Research Part A., vol. 104, no. 1, pp. 113-123, 2016.

[109] D. Codoni, J. Cowan, J. Bradley, W. J. McAuley, M. A. O'Connell, and S. Qi, "Disc-shaped polyoxyethylene glycol glycerides gel nanoparticles as novel protein delivery vehicles," International Journal of Pharmaceutics, vol. 496, no. 2, pp. 1015-1025, 2015.

[110] N. Wang, Y. Li, Y. Zhang, Y. Liao, and W. Liu, "High-strength photoresponsive hydrogels enable surface-mediated gene delivery and light-induced reversible cell adhesion/detachment," Langmuir, vol. 30, no. 39, pp. 11823-11832, 2014.

[111] B. Boonkaew, M. Kempf, R. Kimble, P. Supaphol, and L. Cuttle, "Antimicrobial efficacy of a novel silver hydrogel dressing compared to two common silver burn wound dressings: Acticoat ${ }^{\mathrm{TM}}$ and PolyMem Silver ${ }^{\circledR}, "$ Burns, vol. 40, no. 1, pp. 89-96, 2014.

[112] E. Pinho, M. Grootveld, G. Soares, and M. Henriques, "Cyclodextrin-based hydrogels toward improved wound dressings," Critical Reviews in Biotechnology, vol. 34, no. 4, pp. 328-337, 2014.

[113] X. Chen, M. Zhang, S. Chen et al., "Peptide-modified chitosan hydrogels accelerate skin wound healing by promoting fibro- blast proliferation, migration, and secretion," Cell Transplantation., vol. 26, no. 8, pp. 1331-1340, 2017.

[114] B. Singh, S. Sharma, and A. Dhiman, "Design of antibiotic containing hydrogel wound dressings: biomedical properties and histological study of wound healing," International Journal of Pharmaceutics, vol. 457, no. 1, pp. 82-91, 2013.

[115] Y. Loo, Y.-C. Wong, E. Z. Cai et al., "Ultrashort peptide nanofibrous hydrogels for the acceleration of healing of burn wounds," Biomaterials, vol. 35, no. 17, pp. 4805-4814, 2014.

[116] D. Singh, A. Singh, and R. Singh, "Polyvinyl pyrrolidone/carrageenan blend hydrogels with nanosilver prepared by gamma radiation for use as an antimicrobial wound dressing," Journal of Biomaterials Science, Polymer Edition., vol. 26, no. 17, pp. 1269-1285, 2015.

[117] S.-M. Lee, N. Han, R. Lee et al., "Real-time monitoring of 3D cell culture using a 3D capacitance biosensor," Biosensors and Bioelectronics., vol. 77, pp. 56-61, 2016.

[118] I. Zhitomirsky, "Electrodeposition of organic-inorganic films for biomedical applications," 224th ECS Meeting, 2013, Hamilton, Ontario, Canada, October 27-November 1, 2013, 2013.

[119] M. S. Belluzo, L. F. Medina, A. M. Cortizo, and M. S. Cortizo, "Ultrasonic compatibilization of polyelectrolyte complex based on polysaccharides for biomedical applications," Ultrasonics sonochemistry., vol. 30, pp. 1-8, 2016.

[120] I. Khimji, E. Y. Kelly, Y. Helwa, M. Hoang, and J. Liu, "Visual optical biosensors based on DNA-functionalized polyacrylamide hydrogels," Methods, vol. 64, no. 3, pp. 292-298, 2013.

[121] M. S. Abdelaty and D. Kuckling, "Synthesis and characterization of new functional photo cross-linkable smart polymers containing vanillin derivatives," Gels., vol. 2, no. 1, p. 3, 2016.

[122] Z. Wang, L. Zhang, and Y. Tian, “A durable non-enzymatic electrochemical sensor for monitoring $\mathrm{H}_{2} \mathrm{O}_{2}$ in rat brain microdialysates based on one-step fabrication of hydrogels," The Analyst, vol. 140, no. 11, pp. 3788-3793, 2015.

[123] J. P. Devadhasan and S. Kim, "An ultrasensitive method of real time $\mathrm{pH}$ monitoring with complementary metal oxide semiconductor image sensor," Analytica Chimica Acta, vol. 858, pp. 55-59, 2015.

[124] J. S. Wolffsohn, S. Mroczkowska, O. A. Hunt, P. Bilkhu, T. Drew, and A. Sheppard, "Crossover evaluation of silicone hydrogel daily disposable contact lenses," Optometry and Vision Science, vol. 92, no. 11, pp. 1063-1068, 2015.

[125] G. Su, T. Zhou, Y. Zhang, X. Liu, and A. Zhang, "Microdynamics mechanism of $\mathrm{D}_{2} \mathrm{O}$ absorption of the poly (2-hydroxyethyl methacrylate)-based contact lens hydrogel studied by two-dimensional correlation ATR-FTIR spectroscopy," Soft Matter, vol. 12, no. 4, pp. 1145-1157, 2016.

[126] S. Mikhalovsky, L. Mikhalovska, W. A. Akande, and S. James, Poly (2-hydroxyethyl methacrylate) macroporous cryogel for extracorporeal medical devices, Nazarbayev University, NurSultan city, Republic of Kazakhstan, 2015.

[127] E. García-Millán, S. Koprivnik, and F. J. Otero-Espinar, "Drug loading optimization and extended drug delivery of corticoids from pHEMA based soft contact lenses hydrogels via chemical and microstructural modifications," International Journal of Pharmaceutics, vol. 487, no. 1-2, pp. 260-269, 2015.

[128] T. Billiet, M. Vandenhaute, J. Schelfhout, S. Van Vlierberghe, and P. Dubruel, "A review of trends and limitations in 
hydrogel-rapid prototyping for tissue engineering," Biomaterials, vol. 33, no. 26, pp. 6020-6041, 2012.

[129] L. E. Bertassoni, M. Cecconi, V. Manoharan et al., "Hydrogel bioprinted microchannel networks for vascularization of tissue engineering constructs," Lab on a Chip., vol. 14, no. 13, pp. 2202-2211, 2014.

[130] P. Matricardi, C. Di Meo, T. Coviello, W. E. Hennink, and F. Alhaique, "Interpenetrating polymer networks polysaccharide hydrogels for drug delivery and tissue engineering," Advanced drug delivery reviews., vol. 65, no. 9, pp. 11721187, 2013.

[131] H. K. Cheung, T. T. Y. Han, D. M. Marecak, J. F. Watkins, B. G. Amsden, and L. E. Flynn, "Composite hydrogel scaffolds incorporating decellularized adipose tissue for soft tissue engineering with adipose-derived stem cells," Biomaterials, vol. 35, no. 6, pp. 1914-1923, 2014.

[132] H. Tan and K. G. Marra, "Injectable, biodegradable hydrogels for tissue engineering applications," Materials., vol. 3, no. 3, pp. 1746-1767, 2010.

[133] P. Gentile, V. Chiono, I. Carmagnola, and P. V. Hatton, “An overview of poly (lactic-co-glycolic) acid (PLGA)-based biomaterials for bone tissue engineering," International journal of molecular sciences., vol. 15, no. 3, pp. 3640-3659, 2014.

[134] J. Van Hoorick, M. Markovic, A. Ovsianikov et al., "Hydrogel-polyester combination scaffolds for tissue engineering purposes," in Conference on Advanced Materials for Biomedical Applications (AMBA 2014), Ghent, Belgium, 2014-11-18.

[135] I. De Marco, L. Baldino, S. Cardea, and E. Reverchon, "Effect of process parameters on cellulose acetate scaffolds morphology obtained by supercritical $\mathrm{CO}_{2}$ phase inversion," in Applied Engineering Sciences: Proceedings of the 2014 AASRI International Conference on Applied Engineering Sciences, CRC Press, p. 135, Hollywood, LA, USA, 2014.

[136] T. N. Vo, S. R. Shah, S. Lu et al., "Injectable dual-gelling cell-laden composite hydrogels for bone tissue engineering," Biomaterials, vol. 83, pp. 1-11, 2016.

[137] K. Ren, C. He, C. Xiao, G. Li, and X. Chen, "Injectable glycopolypeptide hydrogels as biomimetic scaffolds for cartilage tissue engineering," Biomaterials, vol. 51, pp. 238-249, 2015.

[138] B. Yang, F. Yao, T. Hao et al., "Development of electrically conductive double-network hydrogels via one-step facile strategy for cardiac tissue engineering," Advanced Healthcare Materials., vol. 5, no. 4, pp. 474-488, 2016. 


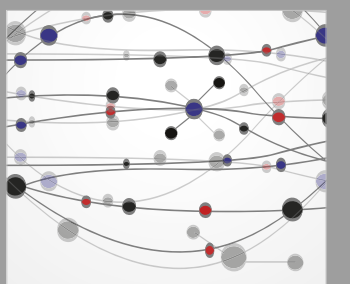

The Scientific World Journal
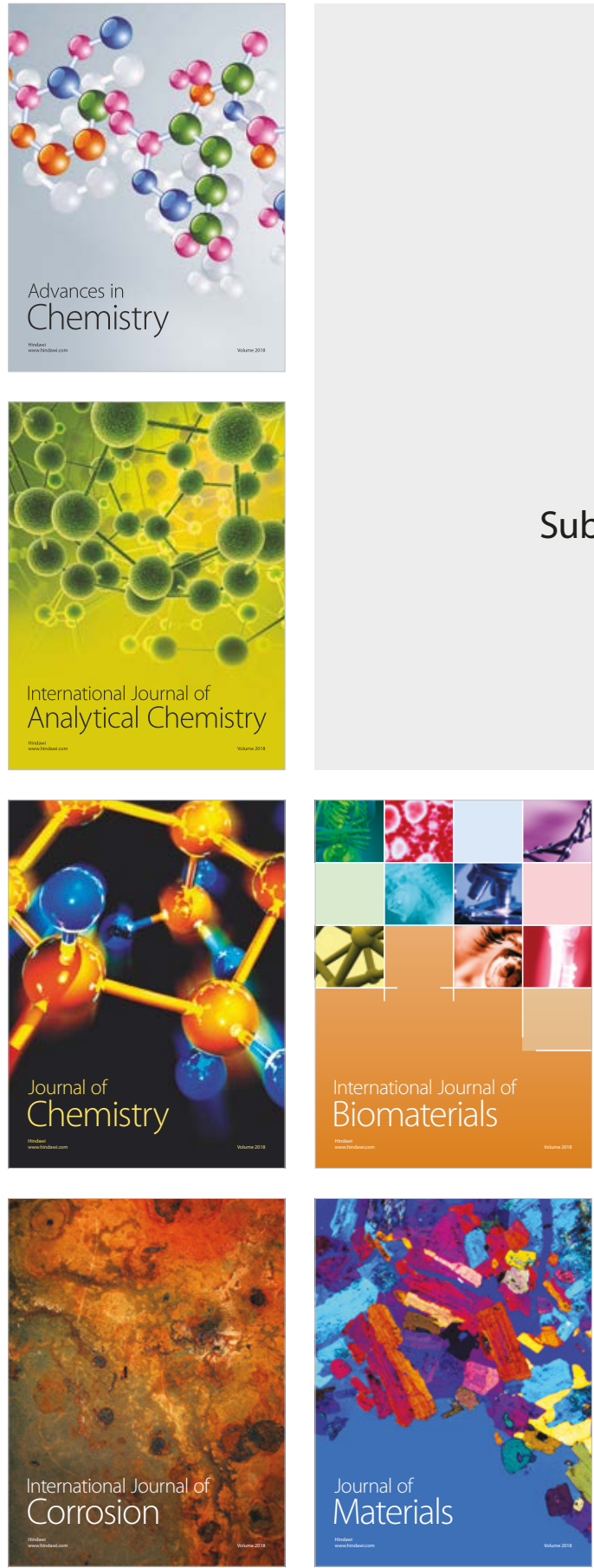

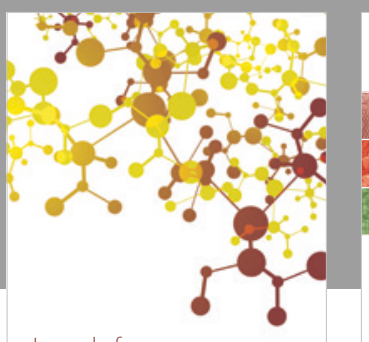

Journal of

Applied Chemistry
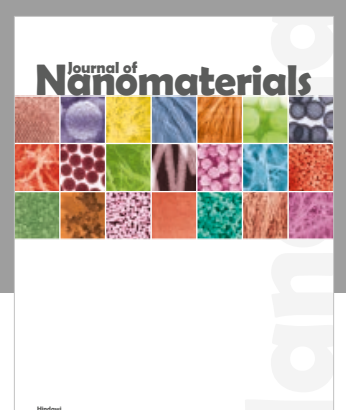

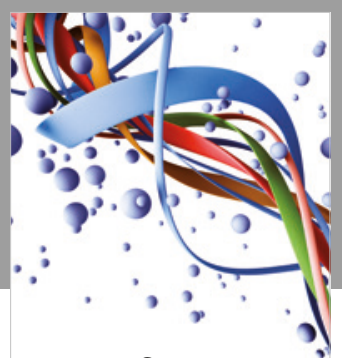

Scientifica

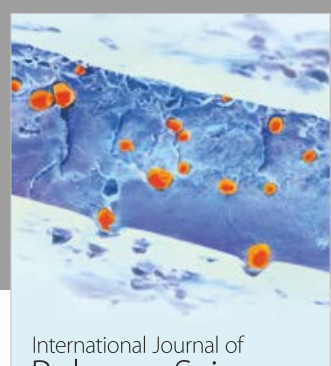

Polymer Science

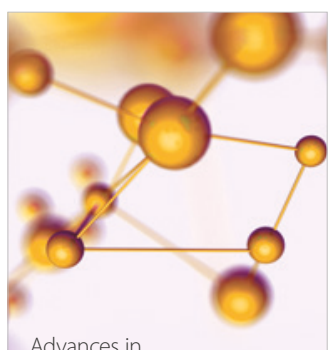

Physical Chemistry
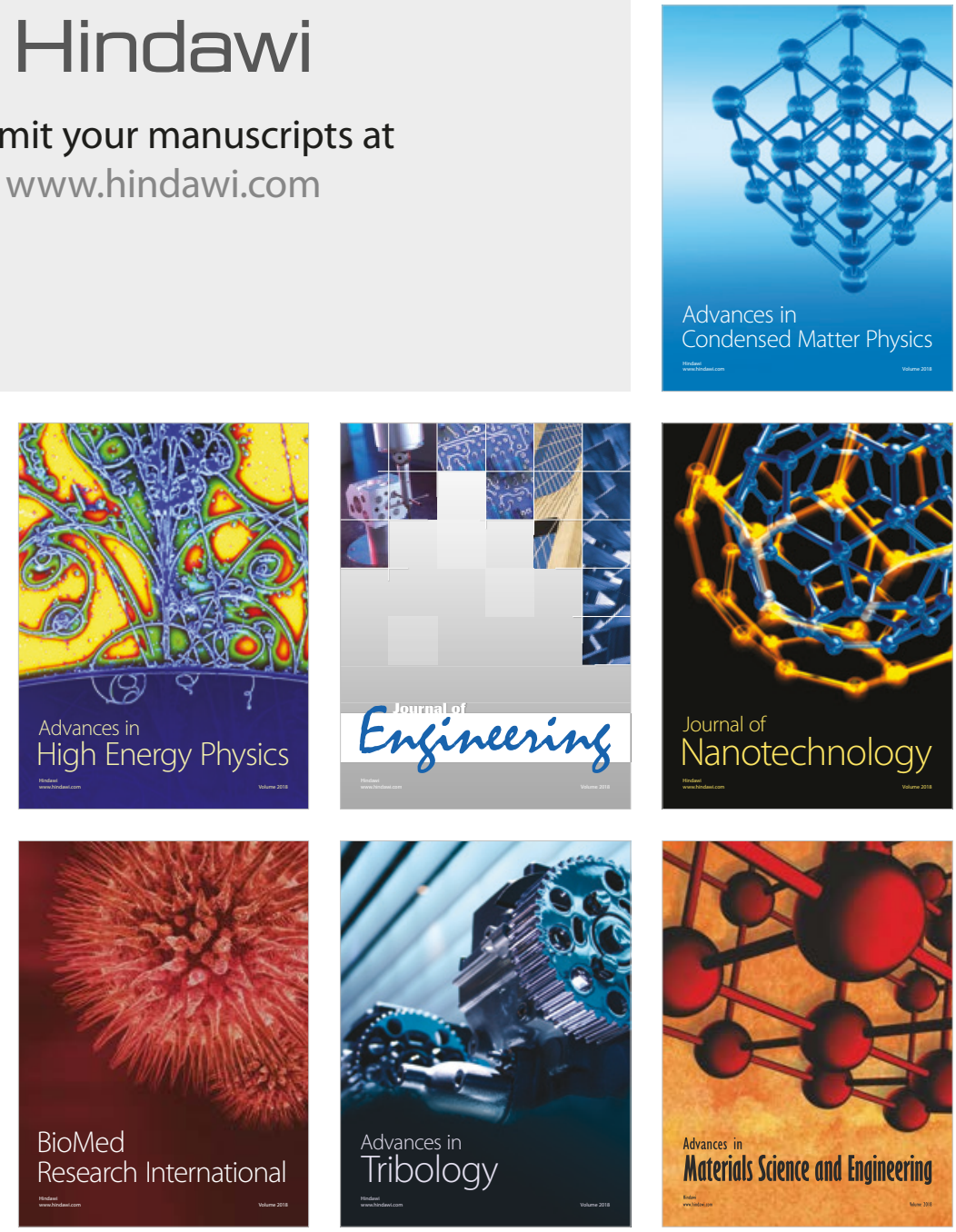